Keywords: Schizoid personality disorder; Loneliness; Biopsychosocial; Cultural.

\title{
Schizoid personality disorder linked to unbearable and inescapable loneliness
}

\author{
Willem H.J. Martens MD, PhD \\ W. Kahn of Theoretical Psychiatry \\ and Neuroscience \\ NETHERLANDS
}

\begin{abstract}
Background and Objectives: More insight is needed into the link between loneliness and schizoid personality disorder in order to construct more adequate diagnostic tools and therapeutic programs.

Methods: A computer-based search of literature (Medline and PsycInfo) between 1970 and 2009.

Results: A combination of intrapsychic, psychosocial, cultural, ethnic, religious, and/or neurobiological factors determine loneliness and associated schizoid etiology. Furthermore, a complex interaction between these influences is prevalent in schizoid etiology.

Conclusion: Loneliness appears to be a crucial factor in the etiology of schizoid personality disorder.
\end{abstract}

Received: 29 May 2009

Revised: 19 September 2009

Accepted: 29 September 2009

\section{Introduction}

According to the DSM-IV-TR " 1 "The essential feature of schizoid personality disorder (SPD) is a pervasive pattern of detachment from social relationships and a restricted range of expression of emotions in interpersonal settings" (p. 694). SPD overlaps with the negative symptoms of schizophrenia: flat affect, lack of motivation, and social withdrawal. SPD have also traits in common with other personality disorder such as lack of empathy with narcissistic (NPD) and antisocial personality disorder (ASPD), withdraw (self-sufficiently in the case of NPD) from others, and failure to form human and social relationships with NPD and avoidant personality disorder.

Persons with SPD are driven into hiding by fear, then experiences a deep, sequestered loneliness that provides the drive to come out of hiding and to go back into the adaptive interface with the world ${ }^{2}$. They tend toward great passivity and look only to 
themselves as sources of validation and enhancement. However, their lack of positive affiliation and affective indifference often put them in a position to be easily taken advantage of by others, and at times they may struggle with personal feelings of social isolation and alienation. At a stylistic level, these persons may seek out and enjoy social and intimate affiliation, but typically not with a great deal of concern ${ }^{3}$.

Millon \& Davis ${ }^{4}$ speculated that the significant deficit in the schizoid disorders is the person's intrinsic incapacities to experience the joyful and pleasurable aspects of life. It does represent a fundamental incapacities to sense the moods and needs that are experienced by others. Klein ${ }^{5}$ suggested that there are at least two quite separate categories of patients with schizoid personality disorder: shy, socially backward, inept, obedient persons who are fearful and therefore isolated but appreciates sociability and would like to be part of the crowd: and there are the asocial, eccentric, (imperceptive and undiplomatic) persons who seek to be alone and have difficulty in relationships with the peers, frequently resulting in social ostraction and scapegoating.

In this article the link between SPD and loneliness is examined, which is relevant to the construction of adequate assessment, diagnostic and therapeutic programs.

\section{Distinctive Determinants of Schizoid Loneliness}

\section{Intrapsychic Mechanisms}

In the first few months of life, anxiety is experienced as fear of persecution, and the infant views the world as either "good" (satisfying, complying, responding, gratifying) or bad (frustrating). The child then proceeds to introject (internalize and assimilate) the good object while keeping out (defending against) the bad objects. The infant projects parts of himself (his organs, his behaviours, his traits) unto the bad object. Splitting allows good to stay separate from bad ${ }^{6}$.

Klein ${ }^{6}$ saw the depressive position as an important developmental milestone that continues to mature throughout the life span. In the depressive position, the infant is able to experience others as whole, which radically alters object relationships from the earlier phase. Klein argued that people who never succeed in working through the depressive position in their childhood will, as a result, continue to struggle with this problem in adult life. For example: the cause that a person may maintain suffering from intense guilt feelings over the death of a loved one, may be found in the unworked- through depressive position. The guilt is there because of a lack of separation between inside and outside and also as a defense mechanism to defend the self against unbearable feelings of intense sadness and sorrow and subsequently the internal object against the unbearable rage of the self, which can destroy the (internal) forever. However, Fairnbairn ${ }^{7}$ argued that early patterns of object relationships can be altered with experience, but often continue to exert a strong influence throughout life ${ }^{7}$.

Kernberg $^{8}$ suggested also that splitting is the major defensive mechanism utilized by the schizoid. Individuals with SPD have difficulties in understanding themselves owing to the conflicting elements of the inner personality. Seen as one variant of the borderline personality organization, their internal worlds, in Kernberg's proposal, are populated by contradictory self-images, one set composed of idealized or frightening aspects of internalized others and another split 
into both shameful and exalted self-images. As a result, there is a persistant state of subjective unreality and identity diffusion, which leads to chronic feelings of emptiness. I suggest that the frightening aspects of internal others will be projected to the external world and might result in a fearful, paranoid attitude and associated social withdrawal and loneliness.

However, other theories suggest that the experience of loss ${ }^{9,10}$ and/or inability to cope with a rejecting mother ${ }^{11}$ might be the core of schizoid development. This loss happens at the time the mother is the infant's sole environment and world, so that it has no alternative defense. The mother is the primary source of security, and the provider of the first relationship, which can counteract the separation-trauma of birth. The disorder represents a failure to resolve interaction, intimacy and attachment conflicts further along in the developmental process, specifically, during separation/individuation subphase $^{9}$. The author suggests that a lasting incapacity to cope with such interaction and attachment conflicts will lead to social isolation and loneliness, which, in turn, will make the existing social-emotional increasingly worse and complicated.

Several psychoanalytic theorists have suggested that emotional deprivation plays a critical role in the development of schizoid personality disorder, which is characterized by an inability to form emotional attachments ${ }^{7,11,12}$. As a consequence of emotional deprivation and an inability to gain security, a lack of satisfaction in interpersonal relationships, and maladaptive schema's and associated cognitive behavior can be observed as components in attachment distortion and painful loneliness that are crucial in schizoid development ${ }^{7,11,12}$.
Laing ${ }^{13}$ suggests that a schizoid individual in one sense is trying to be omnipotent by enclosing within his own being, without recourse to a creative relationship with other, modes of relationship that require the effective presence to him of other people and of the outer world. The imagined advantages are safety for the true self, isolation and hence freedom from others, self-sufficiency, and control (p. 75). As a result, the schizoid becomes fearful of crowds, as they force upon her the recognition that others exist. A way to escape it might be by becoming an aggressor. The author suggests that aggression in persons with SPD might contribute to a new construction of self (more visible as a result of enhanced assertive, extrovert, direct and confronting attitude) in an unconscious attempt to become more interesting and colourful for other people. Aggression can be considered in this way as an effort to overcoming dullness and loneliness. An expression of aggressive resistance could be interpreted as assertive behaviour, refusal to remain an outcast (that is normally absent in schizoid persons) and opportunity to rigorous transformation, and it might be an important step of "being in the world" and becoming released from loneliness.

Withdrawal serves to protect the schizoid individual in the face of psychological collapse. Caught between external and internal conflicts, the person may withdraw in to primitive protective method of autistic encapsulation, and life is endured in a state of isolation, ambivalence, and confusion ${ }^{14}$. Nevertheless, the schizoid persons feels an intense need of intimacy, but the intrapsychic conflicts that inhibit the development of intimacy, are a fear of fusion, a fear of object loss, paranoid-schizoid anxieties, and sexual anxieties ${ }^{15}$. Guntrip ${ }^{11}$ suggests that the early childhood experiences of schizoids often are marked by alternating experiences 
of intrusion and abandonment. The legacy for the child is that his life force threatens mother, which is equivalent to the child experiencing that his life threatens his life. The child copes with this situation by splitting the self; the person is left with a deep and painful intimacy-hunger, dread, and isolation ${ }^{16}$. Furthermore, schizoid people believe that their feelings of love destroy the other and/or lead to their own destruc$\operatorname{tion}^{7,17,18}$. The author suggests that as a consequence this ambiguity the person could conclude that the less painful solution is being alone in order to avoid painful social interactional ambivalence.

The author believes that schizoid condition can be considered as an intrapsychic constellation of oversensitivity, paralysis and paradoxical conflicts (for example fear of as well as hunger for affection and intimacy) as a result of social/emotional rejection; neglect; bad influences; traumatic experience; conflicts; envy; shame; self-hate; low self-esteem (because of their failure to successful development, interactions, socialization and loneliness) rather than indifference to social interactions. An endurable combination of deep suffering and social isolation makes the schizoid development more and more persistent and deep-anchored.

\section{Psychosocial Determinants-Poor Parenting, Neglect, Rejection and Abuse of Children}

Poor parenting might have a strong, lasting, negative impact on the social-emotional, cognitive and moral development of the child. Johnson et al. ${ }^{19}$ revealed in their sample of 593 families that problematic parental behavior (harsh punishing, poor parental supervision, verbal abuse) in the home during the child-rearing years was associated with elevated risk for offspring PD at mean ages of 22 and 33 years. Low parental affection or nurturing was associated with elevated risk for offspring schizoid ${ }^{19}$. In a sample (793 mother and offspring from New York follow-up 18 years from age 5-22) of youths who experienced childhood verbal abuse had elevated SPD symptom levels during adolescence and early adulthood after the covariates were accounted for ${ }^{20}$. The author suggests that physical, social and verbal abuse may provoke in the already vulnerable and shy child strong feelings of being unlovable, inferiority, shame (and linked self-hate) and frustration. This might bring about attachment and associated social interactional problems which, in turn, could contribute to loneliness and SPD etiology.

Childhood sexual abuse in male $(\mathrm{n}=200)^{21}$ female $(\mathrm{n}=88)^{22}$ and various mixed populations ${ }^{23,24}$ correlates with higher scores on the schizoid personality disorder scales of the DSM-III- $\mathrm{R}^{25}$ and DSM-IV Personality Disorder Questionnaire ${ }^{1}$. Bernstein et al. ${ }^{26}$ revealed in his empirical study that emotional abuse and emotional neglect in substance-abusing patients $(n=193$; age 18-60) was related to the traits of DSM-III-R SPD ${ }^{25}$, which formed its own subcluster.

Sexual and emotional abuse/neglect might cause deep feelings of inner emptiness and a blurred and/or confused identity that can be observed in many patients with SPD ${ }^{18}$. Martens ${ }^{27}$ revealed that sexual abuse and emotional abuse/neglect is related to trauma, low self-esteem, self-hate, social withdrawal and maladjustment, social-emotional incapacities, avoidance coping, and neurobiological dysfunctions which might be all determinants of $\mathrm{SPD}^{28}$.

Sexual, physical and emotional abuse and associated severe trauma will likely result in loneliness, because the emotional suffering 
causes a gap between victim and other persons (which brings about loneliness). This might have various reasons such as a) other people's lack of understanding of the pain and maladjusted behavior of the person who suffers; b) fear of confrontation with such pain; c) inadequate responses (too emotional or too rational); d) providing of pushing advices in order to stimulate the patient to become normal.

\section{Cultural, Ethnic and Religious Correlates}

The cultural values, cultural institutions and culture itself are interwoven with our life and correlates with well-being and mental health. Cultural context plays an important role in the development of individual social and behavioral characteristics and peer relationships ${ }^{29}$. The author follows Schwartz \& Pantin ${ }^{30}$ and Bonovitz ${ }^{31}$ who point out that intrapsychic processes are embedded in and interact with relational, social and cultural (and religious) contexts/ dimensions, which are connected with individual intrapsychic processes through dialogue ${ }^{32}$, non-verbal, symbolic and unconscious influences ${ }^{30,31}$.

Because humans need both autonomy and interdependence, persons with either an extreme collectivist orientation (allocentrics) or extreme individualist values (idiocentrics) may be at risk for possession of some features of psychopathology. Caldwell-Harris $\&$ Aycicegi $^{33}$ revealed that for students residing in a highly individualistic society (Boston), collectivism and individualism were not related to DSM-IV SPD ${ }^{1}$. A different pattern was obtained for students residing in a collectivist culture, Istanbul. Here individualism was positively correlated with scales of schizoid personality disorder. Gun- salus \& Kelly ${ }^{34}$ found that Korean students scored significant higher on the schizoid personality scales ${ }^{35}$ compared with American students, and Iwamasa et al. ${ }^{36}$ revealed that criteria for DSM-III-R schizoid PD ${ }^{25}$ was assigned to Asian Americans. The author suggests that the increased risk for Asian Americans could be the result of belonging tot a collectivistic culture, while living in an individualistic society. The author speculates that social expectations in persons with individualistic attitude (when a collective attitude is required) might lead to social exclusion, loneliness and associated SPD. The higher SPD scores of Korean students could be explained by cultural determined personality traits such as introversion, modesty, and social withdrawal.

Viewed through the lens of a cross-level biocultural co-constructive framework, human development is co-constructed by biology and culture through a series of reciprocal interactions between developmental processes and plasticity at different levels ${ }^{37}$. In their review Peterson $\&$ Reiss ${ }^{38}$ analyzed recent cognitive, neuroanatomic, and functional neuroimaging results and concluded that formal education (and upbringing) influences important aspects of the human brain. This provides strong support for the idea that the brain is modulated by literacy and formal education, which in turn change the brain's capacity to interact with its environment, including the individual's contemporary culture ${ }^{38}$. Abnormalities in this complicates neuro-psycho-cultural developmental process could contribute in the etiology of SPD, social exclusion and loneliness.

\section{Neurobiologic Dimension}

Prenatal caloric malnutrition, low birth weight, and prematurity increase (and asso- 
ciated neurodevelopmental aversity) the risk for schizoid personality disorder ${ }^{39}$. Reduced serotonergic (5-HT) function and elevated testosterone have been reported in aggressive populations ${ }^{40}$. Dolan et al. ${ }^{40}$ revealed in persons with DSM-III-R SPD ${ }^{24}$ enhanced 5HT function (prolactin response to d-fenfluramine) and low testosterone concentrations compared with the individuals with psychopathy. Martens ${ }^{41}$ revealed that aggressive attitude in persons with severe personality disorders was linked to reduced capacities to solve problems in a social acceptable way, social exclusion/ rejection and associated loneliness.

Stronger schizoid personality traits in borderline patients $(n=30)$ were significantly related (SCID-II) to reduced leftward parietal cortex asymmetry (using structural magnetic resonance imaging; 3D-MRI $)^{42}$. It is unclear how this abnormality (is linked to visual control of action and representation of spatial information) interferes seriously with normal social-emotional development/awareness and adjustment and how it will contribute to social withdrawal and isolation. The author speculates that a lack of visual control of action might lead to insecurity, low self-esteem and social withdrawal/isolation.

Koponen et al. ${ }^{43}$ evaluated over a period of 30 years the occurrence of psychiatric disorders in patients $(n=60)$ who had experienced a traumatic brain injury and found that $6,7 \%$ $(n=4)$ developed a schizoid personality disorder. It was not discussed by Koponen et al. ${ }^{43}$ which lesions were related to schizoid development. But, it is most likely that lesions in the frontal lobe area (adequate social functioning $)^{44}$, the limbic system (is involved in processing and perception of emotions) ${ }^{44}$ and parietal lobes are involved in lasting social-emotional incapacities, social interactional and attachment problems, loneliness and subsequent schizoid development.

\section{Conclusions}

A combination of and interplay between intrapsychic, psychosocial, cultural and neurobiological aversive factors might interfere with healthy social-emotional (interactional and attachment abilities) and character development. Underdevelopment might bring about social isolation and accustomization to loneliness and lack of social-emotional interactions and training of crucial social kills. This, in return, could result in incapacity to recognize and send subtle social-emotional signs (verbal and non-verbal) which are very important for precise anticipation and understanding during social interactions ("read between the lines" and comprehend the unspoken message) and to avoid miscommunication and associated problems. As a consequence persons with SPD will feel redundant and try to hide themselves. Persons with SPD are convinced (because they were often neglected, rejected and cast-out by other people) that their life is safer and even more comfortable without intensive communication and bonds with others. They have experienced that their attempts to social interactions always lead to troubles, frustrations, and shame. Therefore they try to avoid complicated social-emotional interactions and further negative experiences and correlated further decrease of their self-esteem. Despite of their learned social indifference they will gradually feel shame and frustration about their lack of social contacts and socialemotional inabilities/underdevelopment.

Because schizoid personality disorder is characterized by multidimensional influences a multicomponential approach it should also find concrete shape in current diagnostic models and treatment approaches. Furthermore, psychiatric, psychotherapeutic, cultural, ethnic, genetic and neurologic professionals should cooperate with 
each other in order to develop effective assessment, diagnostic and treatment programs for patients with SPD.

It might be possible that the presented cluster of risk factors is incomplete and that other dimensions such as physical diseases (social isolation as a consequence of long lasting and severe disease), geography and climate (lack of social interactions as a result of sparse population and harsh conditions), social-economic status (social isolation as a result of social rank), and marital status also contribute to schizoid etiology. More research is needed in order to get more insight in the multidimensional dynamics of SPD and to construct more adequate assessment and therapeutic models and programs.

\section{References}

1. American Psychiatric Association. Diagnostic and statistical manual of mental disorders, $4^{\text {th }}$ ed. text rev. (DSM-IV TR). Washington: APA; 2000.

2. Erskine RG. The schizoid process. Transact Anal J 2001; 31(1): 1-7.

3. Rasmussen PR. The Schizoid Prototype. In: Rasmussen PR, ed. Personality-guided cognitive-behavioral therapy. Washington, DC, US: American Psychological Association; 2005. p. 73-87.

4. Millon T, Davis RD. Schizoid personality disorders: The asocial pattern. In: Millon T, Davis RD, eds. Disorders of personality DSM-IV TM and Beyond. New York: Wiley \& Sons; 1996.

5. Klein DF. Psychotropic drugs and the regulation of behavior at activation in psychiatric illness. In: Smith WL, ed. Drugs and cerebral function. Springfield, IL: Thomas; 1970.

6. Klein M. Notes on some schizoid mechanisms. J Psychother Pract Res 1996; 5(2): 164-179.

7. Fairbairn WRD. Psychoanalytic Studies of the Personality. London: Routledge and Kegan Paul; 1952.

8. Kernberg OF. Borderline personality organization. J Am Psychoanal Ass 1967; 15: 641-685.

9. Mahler M, Pine F, Bergman A. The Psychological Birth of the Human Infant: symbiosis and individuation. New York: Basic Books, Inc; 1975.
10. Waska RT. Primitive experiences of loss: Working with the paranoid-schizoid patient. London: Karnac Books; 2002 .

11. Guntrip H. A study of Fairbairn's theory of schizoid reactions. In: Kets de Vries MFR, Perzow SM, eds. Handbook of character studies: Psychoanalytic explorations. Madison, CT, US: International Universities Press, Inc; 1991. p. 407-436.

12. Winnicott DW. The maturational processes and the facilitating environment: Studies in the theory of emotional development (Part 1: Papers). Madison, CT: International Universities Press; 1965.

13. Laing RD. The embodied and unembodied self. In: The divided self. New York: Penguin; 1969. p. 65-77.

14. O'Reilly-Knapp M. Between two worlds: The encapsulated self. Transact Anal J 2001; 31(1): 44-54.

15. Alperin RM. Barriers to intimacy: An object relations perspective. Psychoanal Psychol 2001; 18(1): 137-156.

16. Yontef G. Psychotherapy of schizoid process. Transact Anal J 2001; 31(1): 7-23.

17. Guntrip H. Schizoid Phenomena Object-Relations and the Self. New York: International Universities Press, Inc; 1969.

18. Seinfeld J. The Empty Core: an object relations approach to psychotherapy of the schizoid personality. New Jersey: Jason Aronson, Inc; 1991.

19. Johnson JG, Cohen P, Chen H, Kasen S, Brook JS. Parenting behaviors associated with risk for offspring personality disorder during adulthood. Arch Gen Psychiatry 2006; 63(5): 579-587.

20. Johnson JG, Cohen P, Smailes EM, Skodol AE, Brown J, Oldham JM. Childhood verbal abuse and risk for personality disorders during adolescence and early adulthood. Compr Psychiatry 2001; 42(1): 16-23.

21. Ruggiero JS. The personality sequelae of child maltreatment in drug and alcohol dependent male veterans. Dissertation Abstracts International: Section B: The Sciences and Engineering 1996; 56(8-B): 4592.

22. Swett C, Halpert M. Reported history of physical and sexual abuse in relation to dissociation and other symptomatology in women psychiatric inpatients. J Interpers Violence 1993; 8(4): 545-555.

23. Beitchman JH, Zucker KJ, Hood JE, da Costa GA, Akman D. A review of short-term effects of child sexual abuse. Child Abuse Negl 1991; 15: 537-556.

24. Beitchman JH, Zucker KJ, Hood JE, da Costa GA, Akman D, Cassavia. A review of long-term effects of child sexual abuse. Child Abuse Negl 1992; 16(2): 101-118. 
25. American Psychiatric Association. Diagnostic and statistical manual of mental disorders, $3^{\text {rd }}$ ed. rev. (DSMI11-R). Washington: APA; 2000.

26. Bernstein DP, Stein JA, Handelsman L. Predicting personality pathology among adult patients with substance use disorders: Effects of childhood maltreatment. Addict Behav 1998; 23(6): 855-868.

27. Martens WHJ. Multidimensional model of trauma and correlated antisocial personality disorder. J Loss Trauma 2005; 10(2): 115-131.

28. Cohen JA, Mannarino AP. Posttraumatic Stress Disorder. In: Ollendick TH, March JS, eds. Phobic and anxiety disorders in children and adolescents: A clinician's guide to effective psychosocial and pharmacological interventions. New York: Oxford University Press; 2004. p. 405-432.

29. Mezzich JE, Kleiman A, Fabrega H, Parron D. Cultural and psychiatric diagnosis: DSM-IV perspectives. Washington: American Psychiatric Association; 1996.

30. Schwartz SJ, Pantin H. Identity development in adolescence and emerging adulthood: The interface of self, context, and culture. In: Prescott AP, editor. The concept of self in psychology. Hauppauge, NY, US: Nova Science Publishers; 2006. p. 45-85.

31. Bonovitz C. Locating Culture in the Psychic Field: Transference and Countertransference as Cultural Products. Contemp Psychoanal 2005; 41(1): 55-75.

32. MacRury I. Institutional creativity and pathologies of potential space. Psychodynamic Pract 2007; 13(2): 119-140.

33. Caldwell-Harris CL, Aycicegi A. When personality and culture clash: The psychological distress of allocentrics in an individualistic culture and idiocentrics in a collectivist culture. Transcult Psychiatry 2006; 43(3): 331-361.

33. Gunsalus AJC, Kelly KR. Korean cultural influences on the Millon Clinical Multiaxial Inventory III. J Ment Health Couns 2001; 23(2): 151-161.

35. Millon T, Millon C, Davis R, Grossman S. Millon Clinical Multiaxial Inventory-III. San Antonio: Pearson education; 2009.

36. Iwamasa GY, Larrabee AL, Merritt RD. Are personality disorder criteria ethnically biased? A card-sort analysis. Cultur Divers Ethnic Minor Psychol 2000; 6(3): 284-297.
37. Li SC. Biocultural Co-Construction of Lifespan Development. In: Baltes PB, Reuter-Lorenz PA, Rosler F, eds. Lifespan development and the brain: The perspective of biocultural co-constructivism. New York: Cambridge University Press; 2006. p. 40-57.

38. Petersson KM, Reis A. Characteristics of Illiterate and Literate Cognitive Processing: Implications of BrainBehavior Co-Constructivism. In: Baltes PB, Reuter-Lorenz PA, Rosler F, eds. Lifespan development and the brain: The perspective of biocultural co-constructivism. New York: Cambridge University Press; 2006. p. 279-305.

39. Casper RC. Nutrients, neurodevelopment, and mood. Curr Psychiatry Rep 2004; 6(6): 425-429.

40. Dolan M, Anderson IM, Deakin JF. Relationship between 5-HT function and impulsivity and aggression in male offenders with personality disorders. Br J Psychiatry 2001; 178: 352-359.

41. Martens WHJ. Psychopathy and maturation. PhDthesis, Tilburg University, the Netherlands. Maastricht: Shaker Publishing; 1997.

42. Irle E, Lange C, Sachsse U. Reduced Size and Abnormal Asymmetry of Parietal Cortex in Women with Borderline Personality Disorder. Biol Psychiatry 2005; 57(2): 173-182.

43. Koponen S, Taiminen T, Portin R, Himanen L, Isoniemi $\mathrm{H}$, Heinonen $\mathrm{H}$, et al. Axis I and II psychiatric disorders after traumatic brain injury: a 30-year follow-up study. Am J Psychiatry 2002; 159(8): 1315-1321.

44. Kandel J, Schwartz J, Jessell T. Principles of neural science, $4^{\text {th }}$ edition. New York: McGraw Hill; 2000.

Address for correspondence:

Willem H.J. Martens MD, PhD

Chair of W. Kahn of Theoretical Psychiatry and

Neuroscience, and advisor Psychiatry of the European

Commission (Leonardo da Vinci)

Address: Het Nateland 1

3911XZ Rhenen

The Netherlands

Phone: 31 (0)317 618708

E-mail: Martens_92@hotmail.com

MartensW2000@yahoo.com 